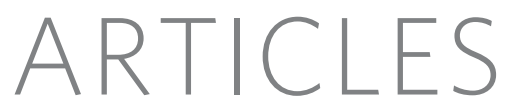

\title{
Efficient tumour formation by single human melanoma cells
}

\author{
Elsa Quintana ${ }^{1 *}$, Mark Shackleton ${ }^{1 *}$, Michael S. Sabel ${ }^{2}$, Douglas R. Fullen ${ }^{3}$, Timothy M. Johnson ${ }^{4} \&$ Sean J. Morrison ${ }^{1}$
}

\begin{abstract}
A fundamental question in cancer biology is whether cells with tumorigenic potential are common or rare within human cancers. Studies on diverse cancers, including melanoma, have indicated that only rare human cancer cells $(0.1-0.0001 \%)$ form tumours when transplanted into non-obese diabetic/severe combined immunodeficiency (NOD/SCID) mice. However, the extent to which NOD/SCID mice underestimate the frequency of tumorigenic human cancer cells has been uncertain. Here we show that modified xenotransplantation assay conditions, including the use of more highly immunocompromised NOD/SCID interleukin-2 receptor gamma chain null $\left(112 \mathrm{rg}^{-/}\right)$mice, can increase the detection of tumorigenic melanoma cells by several orders of magnitude. In limiting dilution assays, approximately $25 \%$ of unselected melanoma cells from 12 different patients, including cells from primary and metastatic melanomas obtained directly from patients, formed tumours under these more permissive conditions. In single-cell transplants, an average of $27 \%$ of unselected melanoma cells from four different patients formed tumours. Modifications to xenotransplantation assays can therefore dramatically increase the detectable frequency of tumorigenic cells, demonstrating that they are common in some human cancers.
\end{abstract}

Traditionally, many cancer cells have been considered to have tumorigenic potential, even though no assay has yet demonstrated that a high percentage of single human cancer cells can form tumours. In contrast, the cancer stem-cell model has suggested that only small subpopulations of cancer cells have tumorigenic potential, based on experiments in which human cancer cells were xenotransplanted into NOD/SCID mice. For example, only one in a million $(0.0001 \%)$ human melanoma cells is tumorigenic in NOD/SCID mice ${ }^{1}$. Indeed, most human cancers have only rare $(<0.1 \%)$ tumorigenic/leukaemogenic cells (also called cancer-initiating cells or cancer stem cells) when transplanted into NOD/SCID or other highly immunocompromised mice ${ }^{1-11}$. Nonetheless, recent studies of mouse haematopoietic malignancies have raised the question of whether NOD/SCID assays underestimate the frequency of human cancer-initiating cells ${ }^{12-14}$. Indeed, human leukaemias exhibit a modestly higher frequency of leukaemogenic cells when assayed in mice that are more highly immunocompromised than NOD/SCID mice ${ }^{15,16}$, although leukaemogenic cells still represent only $1 \%$ of cells in one such model ${ }^{17}$. The critical question is whether optimization of xenotransplantation assays could reveal that some human cancers actually have very common cells with tumorigenic potential despite only having rare tumorigenic cells in NOD/SCID mice.

The question of whether cells with tumorigenic potential are common or rare within human cancers has fundamental implications for therapy. If tumorigenic cells represent small minority populations, as suggested by the evidence supporting the cancer stem-cell model, improved anticancer therapies may be identified based on the ability to kill these cancer stem cells rather than the bulk population of non-tumorigenic cancer cells $^{18,19}$. Alternatively, if cells with tumorigenic potential are common, it will not be possible to treat cancer more effectively or to understand cancer biology better by focusing on small minority subpopulations.

\section{Melanoma-initiating cells are rare in NOD/SCID mice}

Melanoma-initiating (tumorigenic) cells were reported to be rare based on the observation that only 1 in 1,090,000 human metastatic melanoma cells formed tumours within 8 weeks of transplantation into NOD/SCID mice ${ }^{1}$. To assess this, we transplanted $10^{2}-10^{7}$ freshly dissociated melanoma cells obtained directly from seven patients subcutaneously into NOD/SCID mice (see Supplementary Table 1 for more information on tumours). Palpable tumours were evident in some mice 8 weeks after injection of cells from four of seven melanomas (Fig. 1A, B). Limiting dilution analysis ${ }^{20}$ indicated that the average frequency of cells that formed tumours within 8 weeks of transplantation into NOD/SCID mice was 1 in 837,000 (Fig. 1C), confirming the published estimate ${ }^{1}$. However, most tumours took more than 8 weeks to develop (Fig. 1A). On average, tumours first became palpable after $11.4 \pm 3.8$ weeks (mean \pm s.d.), or $14.3 \pm 7.6$ weeks for tumours that arose from fewer than 10,000 injected cells. Variability was high, but the average frequency of cells that formed tumours within 32 weeks was 1 in 111,000 (Fig. 1C; $P<0.0001$ ). The frequency of melanoma-initiating cells is therefore significantly underestimated when tumour formation is monitored for only 8 weeks.

\section{Modified assay reveals more tumorigenic cells}

Some normal human haematopoietic cells engraft more efficiently in NOD/SCID mice lacking the interleukin-2 gamma receptor (NOD/ SCID Il2 $\mathrm{rg}^{-1-}$ ) compared with NOD/SCID mice, owing in part to the lack of natural-killer cell activity in NOD/SCID $\mathrm{Il}_{2 g^{-1-}}$ mice ${ }^{21-24}$. NOD/SCID $I l 2 \mathrm{rg}^{-1-}$ mice have also been used to study cancer arising from human cell lines ${ }^{25,26}$ or human leukaemias ${ }^{15,27}$. We thus compared human melanoma growth in NOD/SCID mice and NOD/SCID Il2 $\mathrm{rg}^{-1-}$ mice to test whether more tumorigenic cells could be detected in more highly immunocompromised NOD/SCID $\mathrm{Il}_{2 \mathrm{rg}^{-1-}}$ mice. Xenografted melanoma cells (human melanomas grown in NOD/SCID mice) from five patients were dissociated, then live human cells were isolated by flow cytometry (excluding mouse haematopoietic and endothelial cells; Fig. 2a) and transplanted sideby-side into NOD/SCID $\mathrm{Il}_{2 g^{-/-}}$and NOD/SCID mice (Fig. 2b). Tumours grew faster in NOD/SCID $\mathrm{Il}_{2} \mathrm{rg}^{-1-}$ mice (Fig. $2 \mathrm{~b}$ and 


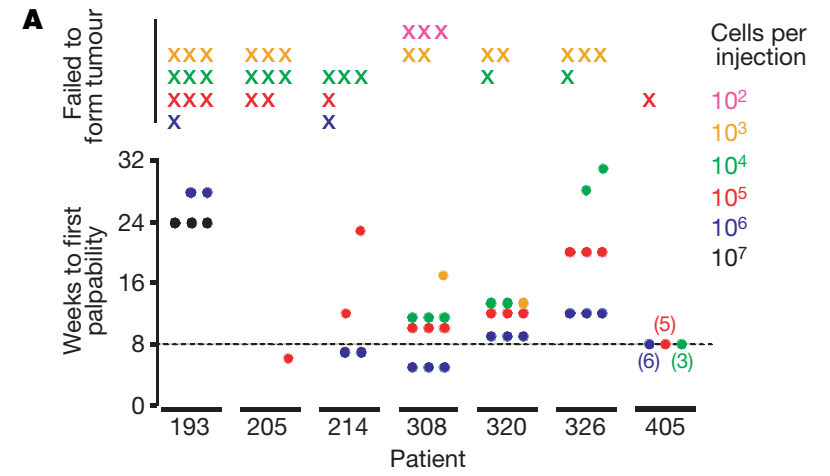

B

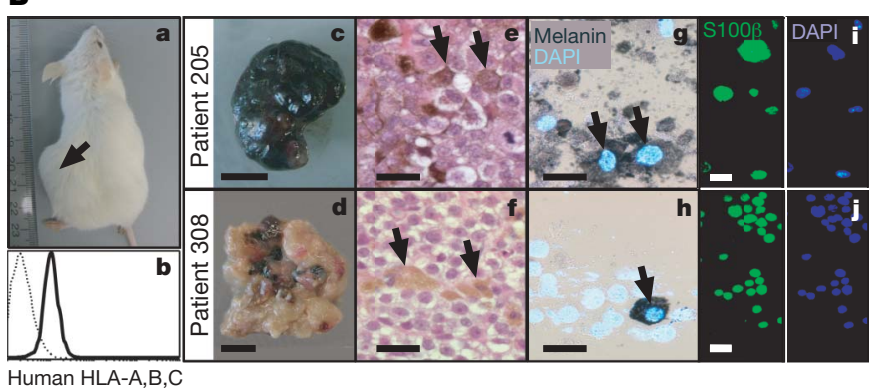

C

\begin{tabular}{l|ll}
$\begin{array}{l}\text { Time after } \\
\text { injection }\end{array}$ & \multicolumn{2}{|l}{$\begin{array}{l}\text { Melanoma-initiating cell frequency } \\
\text { (95\% confidence interval) }\end{array}$} \\
\hline 8 weeks & $1 / 837,000$ & $(1 / 512,000-1 / 1,370,000)$ \\
32 weeks & $1 / 111,000$ * & $(1 / 67,000-1 / 185,000)$
\end{tabular}

Figure 1 | Only rare human melanoma cells form tumours in NOD/SCID mice. A, Tumour development after subcutaneous injection of unfractionated primary melanoma cells directly from seven patients into NOD/SCID mice. Dots represent the times after injection at which individual tumours were first palpable and are coloured according to cell dose. Crosses are injections that failed to form tumours. Dotted line indicates 8 weeks after injection. B, All tumours were diagnosed as metastatic melanoma by clinical pathology (see Supplementary Table 1 for more information). The tumours that formed in mice (a, arrow) became large, grew quickly once they were palpable and were histologically similar to the patient tumours from which they were derived. Flow cytometry demonstrated that most tumour cells expressed human HLA (b; dotted line represents unstained control). Some tumours were highly pigmented

(c) whereas others contained variable pigmentation (d) or were amelanotic (scale bar, $1 \mathrm{~cm}$ ). Sections stained with haematoxylin and eosin through the same tumours showed pigmented cells (e, f, see arrows; bars, $25 \mu \mathrm{m}$ ).

Cytospun cells contained melanin, as indicated by Fontana-Masson staining (g, h, arrows; bars, $25 \mu \mathrm{m}$ ), and showed widespread S100 $\beta$ staining $(\mathbf{i}, \mathbf{j})$, a marker used to diagnose melanoma ${ }^{40}$. C, Limiting dilution analyses of the frequency of tumorigenic melanoma cells in Fig. 1A at 8 weeks or 32 weeks after transplantation $(* P<0.0001)$.

Supplementary Fig. 1), and an increased $(P<0.05)$ frequency of melanoma-initiating cells was observed in NOD/SCID Il2rg ${ }^{-1-}$ mice compared with NOD/SCID mice in every tumour tested (Supplementary Fig. 2). Two melanoma specimens obtained directly from patients ( 465 and 481$)$ also exhibited a significantly $(P<0.05)$ higher frequency of melanoma-initiating cells in NOD/SCID Il2rg ${ }^{-1-}$ mice (Supplementary Fig. 2).

To test whether NOD/SCID Il2 $\mathrm{rg}^{-1-}$ mice selected for growth of more aggressive clones, we transplanted cells from tumours grown in NOD/SCID Il2rg ${ }^{-1-}$ mice back into NOD/SCID versus NOD/SCID $I l 2 \mathrm{rg}^{-1-}$ recipients. The frequency of detectable tumorigenic cells in these tumours went back down in NOD/SCID mice (Supplementary Fig. 3a), demonstrating that heritable changes in the frequency of melanoma-initiating cells did not occur as a result of having grown in NOD/SCID Il2 $\mathrm{rg}^{-1-}$ mice. An increased xenogeneic immune response probably contributes to reduced tumorigenesis by melanoma cells in NOD/SCID mice compared with NOD/SCID Il2rg ${ }^{-1-}$ mice.
We next tested whether further improvements could be made in melanoma cell engraftment. Co-injection with Matrigel ${ }^{28}$ increases tumour formation by cancer cell lines ${ }^{29,30}$ and enhances the engraftment of primary human epithelial cancer cells in immunocompromised mice $^{6,31}$. To test the effect of Matrigel on the ability to detect tumorigenic melanoma cells, we isolated live human melanoma cells by flow cytometry (Fig. 2a) from xenografts derived from three patients. The same cell preparations were transplanted into NOD/SCID $\mathrm{Il}_{2} \mathrm{rg}^{-1-}$ mice after mixing with either vehicle (see Methods) or vehicle with Matrigel. Tumour cells injected with Matrigel produced tumours that grew faster than cells injected with vehicle alone (Fig. $2 \mathrm{c}$ and Supplementary Fig. 4a), and limiting dilution analysis revealed that in every case more melanoma cells were tumorigenic in Matrigel (Supplementary Fig. 4b).

To quantify the combined effect of the individual assay improvements described above, we performed side-by-side transplantation of live melanoma cells isolated by flow cytometry from xenografted tumours from three different patients. The same cell preparations were either mixed with vehicle and injected into NOD/SCID mice or mixed with Matrigel and injected into NOD/SCID $\mathrm{Il}_{2 g^{-1-}}$ mice. In every case, we observed much higher frequencies of tumorigenic cells in the NOD/SCID Il2rg ${ }^{-1-}$ mice. On average, more than 5,000-fold more cells exhibited tumorigenic activity under the modified assay conditions, in which an average of 1 in 9 human melanoma cells formed tumours (Fig. 2d). These results indicate that xenotransplantation assays can be modified to detect much higher than expected frequencies of human cells with tumorigenic potential.

\section{Tumorigenic potential is common in melanoma}

To ensure that our modified assay conditions did not somehow confer tumorigenic capacity on normal human cells, we injected 10,000 primary human melanocytes and/or 100,000 primary human mesenchymal stem cells with Matrigel into NOD/SCID $\mathrm{Il}_{2} \mathrm{rg}^{-1-}$ mice. These injections did not lead to the formation of tumours (Fig. 3a). The frequency of tumorigenic melanoma cells also did not increase with passaging in mice (Supplementary Fig. 4c). Therefore, this xenotransplantation assay does not confer tumorigenic potential on human cells. Consistent with this, serial transplantation experiments demonstrated that the increased tumorigenicity of melanoma cells in NOD/SCID $\mathrm{Il}_{2 \mathrm{rg}^{-/-}}$mice did not reflect a heritable change in the transplanted cells themselves (Supplementary Fig. 3a).

To determine whether xenografted melanomas consistently exhibit high frequencies of tumorigenic cells, we tested five additional tumour samples in an independent series of experiments, including some tumours that exhibited rare tumorigenic cells in NOD/SCID mice (Fig. 1). In each case, live human melanoma cells were isolated by flow cytometry (Fig. 2a) and injected with Matrigel into NOD/SCID Il $2 \mathrm{rg}^{-/-}$mice. Palpable tumours from the injection of eight cells were first detected $10 \pm 2$ weeks after injection. Every tumour exhibited a high frequency of tumorigenic cells (range 1 in 21 to 1 in 5 cells), averaging 1 in 9 cells (Fig. 3b).

To assess whether melanoma cells obtained directly from patients also contain a high frequency of cells with tumorigenic potential, we assessed cells obtained from six patients. These included four metastatic melanomas and two primary cutaneous melanomas. Live melanoma cells were isolated by flow cytometry, excluding human haematopoietic $\left(\mathrm{CD} 45^{+}\right.$or glycophorin $\left.\mathrm{A}^{+}\right)$and endothelial cells $\left(\mathrm{CD} 31^{+}\right)$, which collectively represented $50 \pm 33 \%$ of cells in tumours obtained directly from patients. These excluded cells were confirmed to be haematopoietic and endothelial cells rather than melanoma cells by microscopy, and were greatly depleted of tumorigenic activity when transplanted (data not shown). The melanoma cells isolated by flow cytometry were mixed with Matrigel and injected into NOD/SCID Il2 $\mathrm{rg}^{-/-}$mice. On average, palpable tumours from the injection of 10 cells were first detected $13 \pm 4$ weeks after injection. Every tumour exhibited a high frequency of 
a

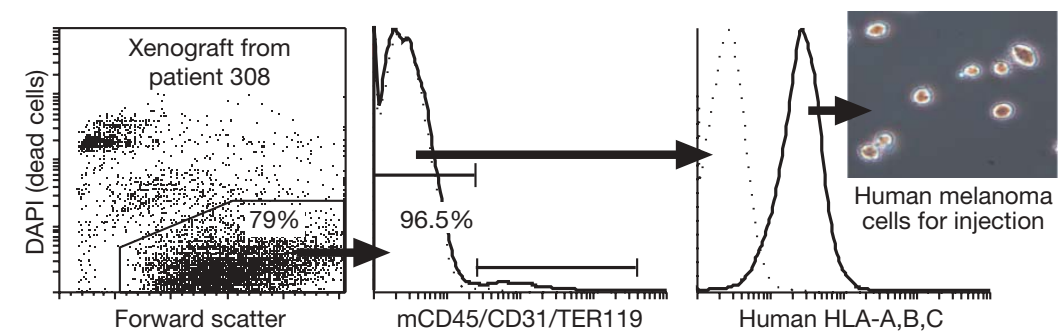

b

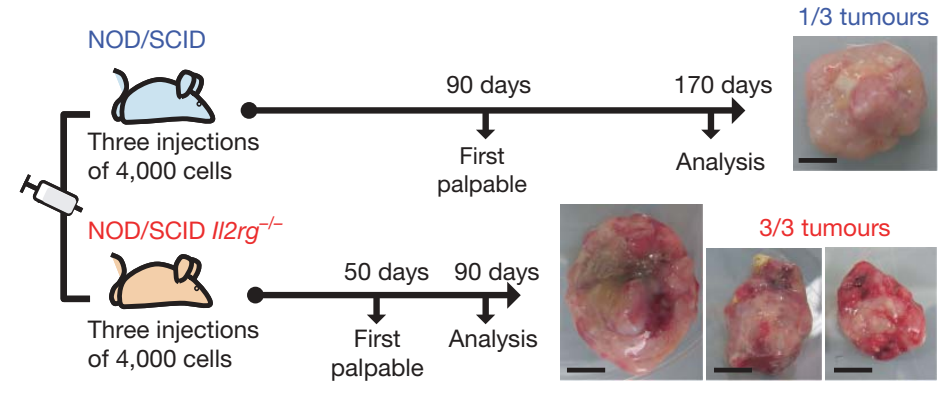

C

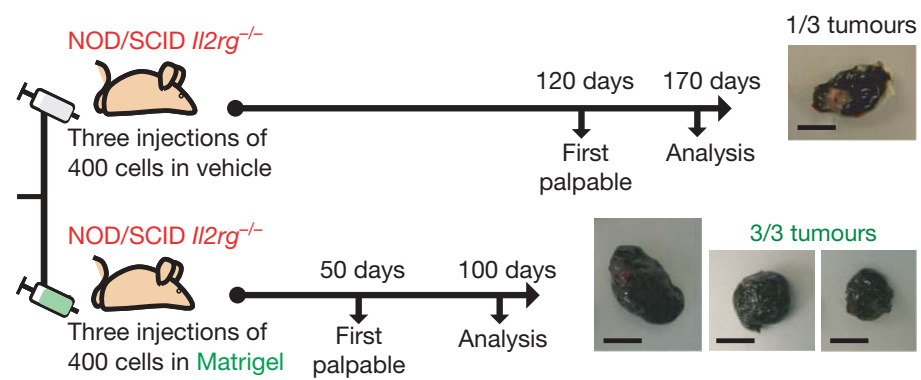

\begin{tabular}{|c|c|c|c|c|c|c|c|c|}
\hline \multirow[t]{2}{*}{ Patient } & \multirow[t]{2}{*}{ Mouse strain } & \multirow[t]{2}{*}{$\begin{array}{l}\text { Co- } \\
\text { injection }\end{array}$} & \multicolumn{4}{|c|}{$\frac{\text { Number of tumours / number of injections }}{\text { cells per injection }}$} & \multicolumn{2}{|c|}{$\begin{array}{l}\text { Melanoma-initiating cell frequency } \\
\text { (95\% confidence interval) }\end{array}$} \\
\hline & & & 50,000 & 5,000 & 50 & 5 & & \\
\hline \multirow[b]{2}{*}{481} & NOD/SCID & Vehicle & $0 / 3$ & $0 / 6$ & $0 / 3$ & & & $(<1 / 60,000)$ \\
\hline & $\mathrm{NOD} / \mathrm{SCID} \| / 2 \mathrm{rg}^{-/-}$ & Matrigel & & & $6 / 6$ & $4 / 6$ & $1 / 5^{\star}$ & $(1 / 2-1 / 13)$ \\
\hline \multirow{2}{*}{491} & NOD/SCID & Vehicle & & $0 / 3$ & $0 / 6$ & & & $(<1 / 5,100)$ \\
\hline & $\mathrm{NOD} / \mathrm{SCID} \| / 2 \mathrm{rg}^{-/-}$ & Matrigel & & & $6 / 6$ & $1 / 6$ & $1 / 15^{*}$ & $(1 / 6-1 / 40)$ \\
\hline \multirow{2}{*}{492} & NOD/SCID & Vehicle & $3 / 3$ & $3 / 6$ & $0 / 6$ & & $1 / 7,300$ & $(1 / 2,400-1 / 22,300)$ \\
\hline & NOD/SCID $/ 12 \mathrm{rg}^{-1-}$ & Matrigel & & & $6 / 6$ & $2 / 6$ & $1 / 11^{*}$ & $(1 / 4-1 / 31)$ \\
\hline \multirow{2}{*}{ All } & NOD/SCID & Vehicle & $3 / 6$ & $3 / 15$ & $0 / 15$ & & $1 / 46,700$ & $(1 / 19,600-1 / 110,900)$ \\
\hline & $\mathrm{NOD} / \mathrm{SCID} / 12 \mathrm{rg}^{-1-}$ & Matrigel & & & $18 / 18$ & $7 / 18$ & $1 / 9^{*}$ & $(1 / 5-1 / 18)$ \\
\hline
\end{tabular}

Figure $2 \mid$ Modifications to the xenotransplantation assay reveal that many more human melanoma cells have tumorigenic potential than detected in NOD/SCID mice.

a, Live human melanoma cells were isolated from xenografted tumours by flow cytometry. After gating to eliminate debris and dead cells, additional gates were drawn to select human $\mathrm{HLA}^{+}$cells and to exclude mouse haematopoietic (CD45 and TER119) and endothelial (CD31) cells (middle panel). The human $\mathrm{HLA}^{+}$cells consistently formed tumours upon transplantation into immunocompromised mice whereas mouse haematopoietic and endothelial cells did not (data not shown). b, Tumour development after side-by-side subcutaneous injections of 4,000 human melanoma cells from a xenograft derived from patient 308 into NOD/ SCID or NOD/SCID Il2 $\mathrm{rg}^{-/-}$mice. c, Tumour development after side-by-side injections of 400 human melanoma cells from a xenograft derived from patient 205 cells into NOD/SCID $\mathrm{Il}_{2 \mathrm{rg}^{-/-}}$ mice, with or without Matrigel. Photographs in b and c show resulting tumours at the time of analysis (bars, $1 \mathrm{~cm}$ ). Similar experiments conducted with xenografted and nonxenografted tumours from several patients demonstrated that a significantly higher frequency of human melanoma cells formed tumours when injected into NOD/SCID $\mathrm{Il} 2 \mathrm{rg}^{-1-}$ mice (Supplementary Fig. 2) or with Matrigel (Supplementary Fig. 4b). The tumours were also palpable earlier and grew faster (Supplementary Figs 1 and 4a).d, Limiting dilution analyses of tumours that arose after 8 weeks in NOD/SCID or NOD/SCID Il2 $\mathrm{rg}^{-1-}$ mice from the side-byside transplantation of melanoma cells (derived from xenografts) mixed with vehicle or Matrigel, respectively. In all cases, melanoma-initiating cells were significantly $\left({ }^{*} P<0.05\right)$ more frequent in the modified assay and represented 1 in 5 to 1 in 15 cells. tumorigenic cells, with an average of 1 in 4 injected cells forming a tumour based on limiting dilution analysis (Fig. 3c). In tumours obtained directly from patients 492, 501 and 509 (the last a cutaneous primary tumour), every injection (6/6) of only 10 cells produced a tumour (Fig. 3c).

We have thus performed injections of small numbers of melanoma cells from 12 different patients and have not yet found a tumour that contained rare tumorigenic cells. Tumorigenic cells were common in all tumours, irrespective of whether they were derived from xenografts or directly from patients, and whether they were from primary cutaneous or metastatic melanomas.

\section{Tumorigenesis by single, unselected melanoma cells}

To our knowledge, no study has yet demonstrated that a high percentage of single cells from a spontaneously occurring human cancer has the potential to form tumours in vivo. To assess this, we sorted live human melanoma cells by flow cytometry from xenografted tumours obtained from four different patients, then deposited one cell per well in Terasaki plates (well volume $10 \mu \mathrm{l}$ ). Wells were visually confirmed to contain a single cell, then mixed with Matrigel and injected into NOD/SCID Il2 $\mathrm{rg}^{-1-}$ mice (Fig. 4a). Depending on the patient, 12-65\% of single cells formed tumours (Fig. 4b). Overall, 69 tumours (27\%) developed from 254 single-cell injections. This demonstrates that xenotransplantation assays can be improved to the point where single human melanoma cells can engraft and form tumours in vivo, confirming that cells with tumorigenic potential are common within human melanomas.

Additional modifications to xenotransplantation assays may further improve the detection of human cells able to form tumours, such that $25 \%$ could remain an underestimate of the percentage of melanoma cells with tumorigenic potential. Because some melanoma cells are fated to undergo cell death or senescence as a result of deleterious genetic changes or proximity to necrotic areas, it is possible that most melanoma cells that are not fated to undergo cell death or senescence have tumorigenic potential. It also remains possible that some melanomas, particularly early-stage primary tumours, may contain less frequent tumorigenic cells than observed in our studies.

\section{Tumorigenic cells are phenotypically heterogeneous}

To assess whether tumorigenic melanoma cells are phenotypically distinct from melanoma cells that fail to form tumours, we examined the expression of more than 50 surface markers on melanomas 
a

\begin{tabular}{lc}
\hline $\begin{array}{l}\text { Injection of normal human cells co-mixed with } \\
\text { Matrigel in NOD/SCID II2rg }\end{array}$ & $\begin{array}{c}\text { Number of tumours/ } \\
\text { number of injections }\end{array}$ \\
\hline 10,000 primary melanocytes & $0 / 6$ \\
100,000 mesenchymal stem cells & $0 / 6$ \\
10,000 melanocytes $+100,000$ mesenchymal stem cells & $0 / 6$ \\
\hline
\end{tabular}

b

\begin{tabular}{|c|c|c|c|c|c|c|}
\hline \multirow[t]{3}{*}{$\begin{array}{l}\text { Tumour } \\
\text { origin }\end{array}$} & \multirow[t]{3}{*}{ Patient } & & $\begin{array}{l}\text { er of tun } \\
\text { er of infe }\end{array}$ & $\begin{array}{l}\text { urs/ } \\
\text { ions }\end{array}$ & \multirow{3}{*}{$\begin{array}{l}\text { Melanoma-initiating } \\
\text { cell frequency (95\% } \\
\text { confidence interval) }\end{array}$} & \multirow{3}{*}{$\begin{array}{c}\text { Weeks } \\
\text { to first } \\
\text { palpability }\end{array}$} \\
\hline & & \multicolumn{3}{|c|}{ Cells per injection } & & \\
\hline & & 200 & 40 & 8 & & \\
\hline \multirow{7}{*}{$\begin{array}{l}\frac{0}{0} \\
\frac{0}{0} \\
\frac{0}{0} \\
\frac{0}{0} \\
\times\end{array}$} & 193 & $12 / 12$ & $12 / 12$ & $8 / 12$ & $1 / 7 \quad(1 / 4-1 / 14)$ & $11 \pm 1$ \\
\hline & 205 & $18 / 18$ & $18 / 18$ & $13 / 18$ & $1 / 6 \quad(1 / 3-1 / 11)$ & $9 \pm 2$ \\
\hline & 214 & $6 / 6$ & $5 / 6$ & $2 / 6$ & $1 / 21 \quad(1 / 9-1 / 48)$ & $9 \pm 0$ \\
\hline & 308 & $11 / 11$ & $11 / 12$ & $10 / 11$ & $1 / 8 \quad(1 / 4-1 / 15)$ & $8 \pm 2$ \\
\hline & 405 & $6 / 6$ & $5 / 6$ & $4 / 6$ & $1 / 14 \quad(1 / 6-1 / 33)$ & $9 \pm 1$ \\
\hline & 481 & $6 / 6$ & $6 / 6$ & $5 / 6$ & $1 / 5 \quad(1 / 2-1 / 12)$ & $11 \pm 0$ \\
\hline & All & $59 / 59$ & $57 / 60$ & $42 / 59$ & $1 / 9 \quad(1 / 7-1 / 12)$ & $10 \pm 2$ \\
\hline
\end{tabular}

c

\begin{tabular}{|c|c|c|c|c|c|c|c|}
\hline \multirow[t]{3}{*}{$\begin{array}{l}\text { Tumour } \\
\text { origin }\end{array}$} & \multirow[t]{3}{*}{ Patient } & \multirow{2}{*}{\multicolumn{3}{|c|}{$\begin{array}{c}\text { Number of tumours/ } \\
\text { number of infections } \\
\text { Cells per injection }\end{array}$}} & \multirow{3}{*}{\multicolumn{2}{|c|}{$\begin{array}{l}\text { Melanoma-initiating } \\
\text { cell frequency (95\% } \\
\text { confidence interval) }\end{array}$}} & \multirow{3}{*}{$\begin{array}{c}\text { Weeks } \\
\text { to first } \\
\text { palpability }\end{array}$} \\
\hline & & & & & & & \\
\hline & & 1,000 & 100 & 10 & & & \\
\hline \multirow{7}{*}{ 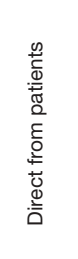 } & 487 & $6 / 6$ & $6 / 6$ & $5 / 6$ & $1 / 6$ & $(1 / 2-1 / 15)$ & $12 \pm 3$ \\
\hline & 491 & $6 / 6$ & $6 / 6$ & $5 / 6$ & $1 / 6$ & $(1 / 2-1 / 15)$ & $12 \pm 3$ \\
\hline & 492 & $6 / 6$ & $6 / 6$ & $6 / 6$ & & $(>1 / 11)$ & $9 \pm 2$ \\
\hline & 501 & $6 / 6$ & $6 / 6$ & $6 / 6$ & & $(>1 / 11)$ & $15 \pm 2$ \\
\hline & 498 (cut) & $6 / 6$ & $6 / 6$ & $5 / 6$ & $1 / 6$ & $(1 / 2-1 / 15)$ & $20 \pm 4$ \\
\hline & 509 (cut) & $6 / 6$ & $6 / 6$ & $6 / 6$ & & $(>1 / 11)$ & $10 \pm 2$ \\
\hline & All & $36 / 36$ & $36 / 36$ & $33 / 36$ & $1 / 4$ & $(1 / 3-1 / 6)$ & $13 \pm 4$ \\
\hline
\end{tabular}

Figure 3 | A high percentage of human melanoma cells are tumorigenic, but normal human cells are not. a, Large doses of primary human melanocytes and/or mesenchymal stem cells were mixed with Matrigel and transplanted into NOD/SCID Il2 $\mathrm{rg}^{-1-}$ mice. No tumours were palpable after 22 weeks. The frequency of melanoma-initiating cells in 12 human tumours obtained either from xenografts (b) or directly from patients (c) was calculated by limiting dilution analysis. Tumours from patients 498 and 509 were primary cutaneous lesions (cut), whereas other tumours were metastases (Supplementary Table 1). In each case, live human melanoma cells were isolated by flow cytometry, mixed in Matrigel and injected subcutaneously into NOD/SCID Il $2 \mathrm{rg}^{-1-}$ mice. Eleven per cent of melanoma cells from xenografts and $25 \%$ of melanoma cells directly from patients formed tumours within 32 weeks of transplantation. Weeks to palpability

(mean \pm s.d.) are indicated for the lowest dose of cells from each tumour.

derived from several patients (Supplementary Table 2). These included markers of other cancer stem-cell populations, melanocytes, melanoma, neural-crest derivatives and other cell types. Fifteen of these markers (A2B5, c-kit, CD44, CD49B, CD49D, CD49f, CD54, CD133, CD166, E-cadherin, HNK-1, L1CAM, MCAM, N-cadherin and p75) were heterogeneously expressed by melanoma cells from multiple patients and were tested for the ability to distinguish tumorigenic from non-tumorigenic melanoma cells in vivo (Supplementary Table 2). In each case, melanoma cells were fractionated by flow cytometry (except CD133, which was sometimes fractionated using magnetic beads as in previous studies ${ }^{10,32}$ ) and cells that expressed different levels of the indicated markers were injected into NOD/SCID $\mathrm{Il}_{2 \mathrm{rg}^{-1-}}$ mice. In every case, tumours arose from all fractions of cells. We found no marker that distinguished tumorigenic from non-tumorigenic cells (Supplementary Table 2). Detailed results are shown for CD49f ( $\alpha_{6}$ integrin, a marker expressed by many different stem cells ${ }^{33}$ ) and L1CAM (which is associated with CD133 expression in glioma stem cells ${ }^{34}$ ) in Supplementary Fig. 5.

A previous study found that 1 in $120,735(0.00083 \%) \mathrm{ABCB}^{+}$ metastatic melanoma cells formed tumours in NOD/SCID mice, a

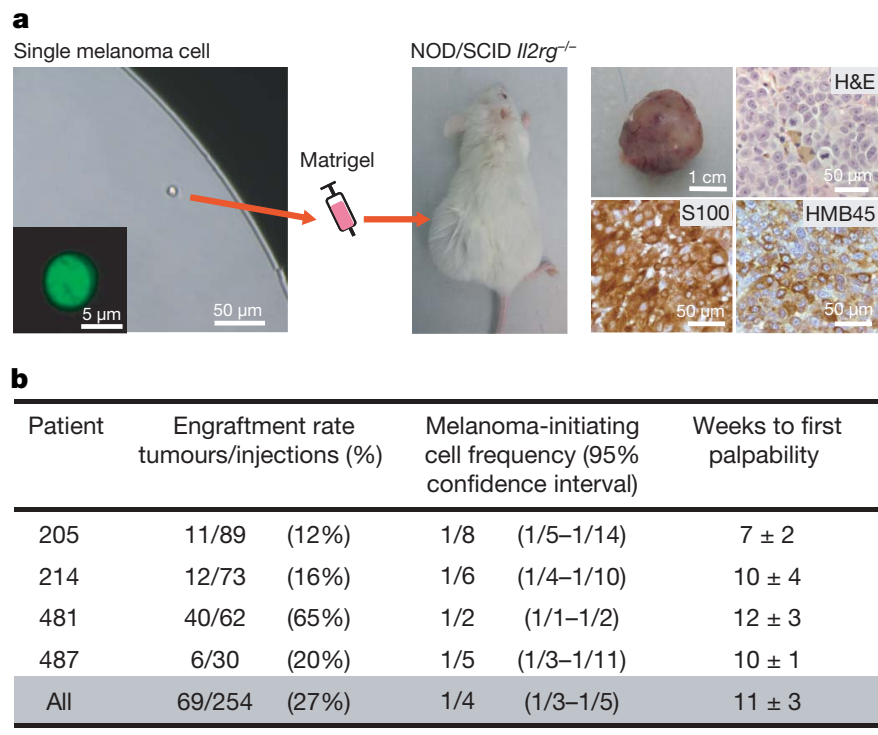

Figure 4 | Efficient tumour development from the xenotransplantation of single human melanoma cells. a, Flow-cytometrically isolated human melanoma cells derived from xenografts from four patients were diluted into Terasaki microwells such that wells containing single cells could be identified by phase contrast microscopy. In control experiments, the presence of single cells was confirmed by the observation of single nuclei with Acridine Orange staining (inset) in 90 out of 90 cases. The single cells were mixed with Matrigel and injected into NOD/SCID $I l 2 \mathrm{rg}^{-l-}$ mice. Tumours arising from the injection of single cells were confirmed to be melanoma by haematoxylin and eosin, S100 and HMB45 staining (right panels show sections from a tumour that arose from a single cell obtained from patient 214). b, The percentage of single-cell injections $(69 / 254=27 \%)$ that formed tumours within 20 weeks of transplantation. Weeks to first palpability (mean \pm s.d.) are indicated for each set of tumours.

tenfold enrichment over unfractionated cells ${ }^{1}$. Because ABCB5 expression has been shown to correlate with the expression of CD166 and CD133 (ref. 35), we tested whether CD166 or CD133 could enrich tumorigenic melanoma cells in the modified xenotransplantation assay (Supplementary Fig. 6). The frequency of $\mathrm{CD}_{13} 3^{+}$cells in tumours from 12 different patients was consistently lower (usually lower than 5\%) than the frequency of tumorigenic cells in the same tumours (5-20\%; Supplementary Fig. 6b). Moreover, CD133 ${ }^{+}$cells were not enriched for tumorigenic melanoma cells. Both $\mathrm{CD}_{133}{ }^{+}$and $\mathrm{CD} 33^{-}$fractions from two different melanomas exhibited very high frequencies of tumorigenic cells (Supplementary Fig. 6c). Flow cytometry indicated that all of the resulting tumours contained both $\mathrm{CD}_{133^{+}}$and $\mathrm{CD} 133^{-}$cells, irrespective of whether they were derived from $\mathrm{CD}_{133^{+}}$or $\mathrm{CD} 133^{-}$cells (data not shown). Both $\mathrm{CD} 166^{+}$and $\mathrm{CD} 166^{-}$fractions also contained very high frequencies of tumorigenic melanoma cells (Supplementary Fig. 6f). We have therefore been unable to identify any phenotypic differences that distinguish tumorigenic from non-tumorigenic melanoma cells. These results raise the possibility that markers that enrich rare cells with tumorigenic potential in NOD/SCID mice may fail to distinguish tumorigenic from nontumorigenic cells in assays that detect much higher frequencies of tumorigenic cells, though more work will be required to test this fully.

\section{Discussion}

Our data demonstrate that modifications in xenotransplantation assays can dramatically increase - by several orders of magnitudethe detectable frequency of cells with tumorigenic potential. This means that some cancers that appear to have rare tumorigenic cells in NOD/SCID mice actually have very common cells with tumorigenic capacity under other conditions. Other cancers may still have infrequent tumorigenic cells, even when studied under optimized conditions, but the frequency and phenotypic diversity of these cells 
may be considerably greater than currently thought. Efforts to optimize the xenotransplantation of human cancer cells will be necessary to identify and study the full spectrum of human cancer cells capable of contributing to disease progression.

It is important to note that neither our study nor previous cancer stem-cell studies have addressed which cells actually contribute to tumour growth and disease progression in patients. These studies address the potential of cancer cells to proliferate extensively and to form tumours/leukaemias, not their actual fate within patients. Depending on how different the tumour environment is within patients compared with mouse models, it is possible that different cancer cells form tumours in mouse models than in human patients. Thus, although we observe a high percentage of melanoma cells that have the potential to proliferate extensively and form new tumours, it is possible that an even greater, or a much smaller, fraction of melanoma cells actually contributes to disease progression in patients.

Although most cancer stem-cell studies have detected only rare cells with tumorigenic capacity, it has recently been pointed out that leukaemogenic/tumorigenic cells do not necessarily have to be rare for cancers to follow a cancer stem-cell model ${ }^{17}$. Our observation that NOD/SCID xenotransplantation can dramatically underestimate the frequency of tumorigenic cells in at least some human cancers does not necessarily mean that such cancers will not have intrinsically different populations of tumorigenic and non-tumorigenic cells. Having said that, the frequency of tumorigenic cells in human melanoma is much higher than reported for any cancer previously suggested to follow a cancer stem-cell model, and we have not yet been able to identify phenotypic differences between melanoma cells that form tumours and those that do not. If markers that distinguish tumorigenic from non-tumorigenic melanoma cells in optimized xenotransplantation assays are identified in future, it will be important to test whether they reflect epigenetic differences between cells (as envisioned under the cancer stem-cell model) or genetic/environmental differences between cells.

Although cells with tumorigenic potential are likely to be much more frequent in most human cancers than estimated based on NOD/SCID transplantation, we believe the available evidence continues to support the conclusion that some human cancers follow a cancer stem-cell model. For example, data from the syngeneic transplantation of mouse acute myeloid leukaemia (AML) ${ }^{36}$ and the transplantation of human AMLs into improved mouse model ${ }^{17}$ continue to suggest that many AMLs have small, intrinsically distinct subpopulations of AML-initiating cells. Extensive clinical experience with germ-cell cancers proves that therapies that eliminate the undifferentiated subset of cancer cells can cure patients, even if differentiated cancer cells are left behind ${ }^{37,38}$. Nonetheless, careful optimization of xenotransplantation assays will probably yield a more nuanced view in which some cancers contain small subpopulations of cancer-initiating cells, whereas others contain common tumorigenic cells with little evidence of hierarchical organization. In both cases it will be critical to identify all cancer cells that have the potential to contribute to disease in patients in order to develop more effective therapies.

\section{METHODS SUMMARY}

Tumour cell preparation. Melanoma specimens were obtained from patients according to protocols approved by the Institutional Review Board of the University of Michigan Medical School (IRBMED approval numbers 20041058 and 2000-0713). Fresh tumour tissue was mechanically dissociated, enzymatically digested and filtered to obtain a single-cell suspension.

Cell labelling and sorting. Cells were stained with directly conjugated antibodies to human CD45, human CD31 and glycophorin A (to eliminate haematopoietic and endothelial cells from tumours obtained directly from patients) or mouse CD45, mouse CD31, Ter119 and human leukocyte antigen (HLA)-A, -B, -C (to eliminate mouse haematopoietic and endothelial cells and select human cells from xenografted tumours). Cells were resuspended in $10 \mu \mathrm{g} \mathrm{ml}^{-1} 4,6$ diamidino-2-phenylindole (DAPI) to label dead cells and sorted on FACSVantage SE or FACSAria flow cytometers. Some samples were labelled with anti-CD133 antibody and separated magnetically using a CD133 Cell Isolation
Kit (Miltenyi Biotec). When testing other markers, cells were usually labelled with unconjugated primary antibodies (see Supplementary Table 2) and then with directly conjugated secondary antibodies before staining with the directly conjugated antibodies described above.

Transplantation of human melanoma cells. After sorting, cells were counted and re-suspended in staining medium ( $\mathrm{L} 15$ medium containing $1 \mathrm{mg} \mathrm{ml}^{-1} \mathrm{BSA}$, $1 \%$ penicillin/streptomycin and 10 mM HEPES ( $\mathrm{pH} 7.4$ )), with or without $25 \%$ Matrigel (BD Biosciences). Subcutaneous injections of human melanoma cells were performed in NOD.CB17-Prkdcscid/J (NOD/SCID) and NOD.CB17Prkdcscid Il2rgtm1Wjl/SzJ (NOD/SCID Il2 $\mathrm{rg}^{-/-}$) mice (Jackson Laboratories) according to protocols approved by the Committee on the Use and Care of Animals at the University of Michigan (protocol number 9055).

Statistics. Limiting dilution analyses were performed based on Bonnefoix et al. ${ }^{39}$, using the limdil function of the 'StatMod' package (author G.K. Smyth, http:// bioinf.wehi.edu.au/software/limdil/), part of the $\mathrm{R}$ statistical software project (http://www.r-project.org). Melanoma-initiating cell frequencies were compared using likelihood ratio tests.

Full Methods and any associated references are available in the online version of the paper at www.nature.com/nature.

\section{Received 21 August; accepted 14 October 2008.}

1. Schatton, T. et al. Identification of cells initiating human melanomas. Nature 451, 345-349 (2008)

2. Li, C. et al. Identification of pancreatic cancer stem cells. Cancer Res. 67, 1030-1037 (2007)

3. Prince, M. E. et al. Identification of a subpopulation of cells with cancer stem cell properties in head and neck squamous cell carcinoma. Proc. Natl Acad. Sci. USA 104, 973-978 (2007).

4. Wu, C. et al. Side population cells isolated from mesenchymal neoplasms have tumor initiating potential. Cancer Res. 67, 8216-8222 (2007).

5. Wang, J. C. et al. High level engraftment of NOD/SCID mice by primitive normal and leukemic hematopoietic cells from patients with chronic myeloid leukemia in chronic phase. Blood 91, 2406-2414 (1998)

6. Al-Haji, M. et al. Prospective identification of tumorigenic breast cancer cells Proc. Natl Acad. Sci. USA 100, 3983-3988 (2003)

7. Cox, C. V. et al. Characterization of acute lymphoblastic leukemia progenitor cells. Blood 104, 2919-2925 (2004)

8. Hope, K. J., Jin, L. \& Dick, J. E. Acute myeloid leukemia originates from a hierarchy of leukemic stem cell classes that differ in self-renewal capacity. Nature Immunol. 5, 738-743 (2004).

9. Dalerba, P. et al. Phenotypic characterization of human colorectal cancer stem cells. Proc. Natl Acad. Sci. USA 104, 10158-10163 (2007).

10. O'Brien, C. A. et al. A human colon cancer cell capable of initiating tumour growth in immunodeficient mice. Nature 445, 106-110 (2007).

11. Ricci-Vitiani, L. et al. Identification and expansion of human colon-cancerinitiating cells. Nature 445, 111-115 (2007).

12. Williams, R. T., den Besten, W. \& Sherr, C. J. Cytokine-dependent imatinib resistance in mouse $\mathrm{BCR}^{-\mathrm{ABL}^{+}}{ }^{+}$, Arf-null lymphoblastic leukemia. Genes Dev. 21, 2283-2287 (2007).

13. Kelly, P. N. et al. Tumor growth need not be driven by rare cancer stem cells. Science 317, 337 (2007).

14. Somervaille, T. C. \& Cleary, M. L. Identification and characterization of leukemia stem cells in murine MLL-AF9 acute myeloid leukemia. Cancer Cell 10, 257-268 (2006).

15. Agliano, A. et al. Human acute leukemia cells injected in NOD/LtSz-SCID/IL- $\gamma^{\text {null }}$ mice generate a faster and more efficient disease compared to other NOD/SCIDrelated strains. Int. J. Cancer 123, 2222-2227 (2008).

16. Feuring-Buske, M. et al. Improved engraftment of human acute myeloid leukemia progenitor cells in $\beta$-2-microglobulin-deficient NOD/SCID mice and in NOD/ SCID mice transgenic for human growth factors. Leukemia 17, 760-763 (2003).

17. Kennedy, J. A. et al. Comment on 'Tumor growth need not be driven by rare cancer stem cells'. Science 318, 1722; author reply 1722 (2007)

18. Pardal, R., Clarke, M. F. \& Morrison, S. J. Applying the principles of stem-cell biology to cancer. Nature Rev. Cancer 3, 895-902 (2003).

19. Wang, J. C. \& Dick, J. E. Cancer stem cells: lessons from leukemia. Trends Cell Biol. 15, 494-501 (2005).

20. Shackleton, M. et al. Generation of a functional mammary gland from a single stem cell. Nature 439, 84-88 (2006).

21. Shultz, L. D. et al. Human lymphoid and myeloid cell development in NOD/LtSzscid IL2R $\gamma^{\text {null }}$ mice engrafted with mobilized human hemopoietic stem cells. J. Immunol. 174, 6477-6489 (2005).

22. Ito, M. et al. NOD $/ \mathrm{SCID} / \gamma_{c}{ }^{\text {null }}$ mouse: an excellent recipient mouse model for engraftment of human cells. Blood 100, 3175-3182 (2002).

23. McKenzie, J. L. et al. Human short-term repopulating stem cells are efficiently detected following intrafemoral transplantation into NOD/SCID recipients depleted of CD122 ${ }^{+}$cells. Blood 106, 1259-1261 (2005).

24. Shultz, L. D. et al. Regulation of human short-term repopulating cell (STRC) engraftment in NOD/SCID mice by host CD122 ${ }^{+}$cells. Exp. Hematol. 31, 551-558 (2003). 
25. Hamada, K. et al. Liver metastasis models of colon cancer for evaluation of drug efficacy using NOD/Shi-scid IL2R $\gamma^{\text {null }}$ (NOG) mice. Int. J. Oncol. 32, 153-159 (2008)

26. Suemizu, H. et al. Identification of a key molecular regulator of liver metastasis in human pancreatic carcinoma using a novel quantitative model of metastasis in NOD/SCID/ $\gamma$ cnull (NOG) mice. Int. J. Oncol. 31, 741-751 (2007).

27. Ishikawa, F. et al. Chemotherapy-resistant human AML stem cells home to and engraft within the bone-marrow endosteal region. Nature Biotechnol. 25, 1315-1321 (2007)

28. Kleinman, H. K. \& Martin, G. R. Matrigel: basement membrane matrix with biological activity. Semin. Cancer Biol. 15, 378-386 (2005).

29. Pretlow, T. G. et al. Transplantation of human prostatic carcinoma into nude mice in Matrigel. Cancer Res. 51, 3814-3817 (1991).

30. Sweeney, T. M. et al. Basement membrane and the SIKVAV laminin-derived peptide promote tumor growth and metastases. Cancer Metastasis Rev. 10, 245-254 (1991).

31. Fridman, R. et al. Enhanced tumor growth of both primary and established human and murine tumor cells in athymic mice after coinjection with Matrigel. J. Natl Cancer Inst. 83, 769-774 (1991).

32. Singh, S. K. et al. Identification of human brain tumour initiating cells. Nature 432 396-401 (2004).

33. I washita, T. et al. Hirschsprung disease is linked to defects in neural crest stem cell function. Science 301, 972-976 (2003).

34. Bao, S. et al. Targeting cancer stem cells through L1CAM suppresses glioma growth. Cancer Res. 68, 6043-6048 (2008)

35. Frank, N. Y. et al. ABCB5-mediated doxorubicin transport and chemoresistance in human malignant melanoma. Cancer Res. 65, 4320-4333 (2005).

36. Yilmaz, O. H. et al. Pten dependence distinguishes haematopoietic stem cells from leukaemia-initiating cells. Nature 441, 475-482 (2006).

37. Horwich, A., Shipley, J. \& Huddart, R. Testicular germ-cell cancer. Lancet 367 754-765 (2006).

38. Kleinsmith, L. J. \& Pierce, G. B. Multipotentiality of single embryonal carcinoma cells. Cancer Res. 24, 1544-1551 (1964).

39. Bonnefoix, T. et al. Fitting limiting dilution experiments with generalized linear models results in a test of the single-hit Poisson assumption. J. Immunol. Methods 194, 113-119 (1996).
40. Nakajima, T. et al. Immunohistochemical demonstration of S100 protein in malignant melanoma and pigmented nevus, and its diagnostic application. Cancer 50, 912-918 (1982)

Supplementary Information is linked to the online version of the paper at wWw.nature.com/nature.

Acknowledgements This work was supported by the Howard Hughes Medical Institute and by the Allen $\mathrm{H}$. Blondy Research Fellowship. The University of Michigan Melanoma Bank was supported by a gift from Lewis and Lillian Becker. Flow cytometry was partly supported by the University of Michigan

Comprehensive Cancer Center grant from the National Institutes of Health

CA46592. We thank: D. Adams, M. White and the University of Michigan Flow Cytometry Core Facility for support; N. McAnsh and the University of Michigan Cancer Centre Histology Core for histological studies; G. K. Smyth for assistance with statistics; and Z. Azizan for support with tissue collection. Antibody production was supported in part by the National Institute of Diabetes, Digestive and Kidney Diseases, grant NIH5P60-DK20572 to the Michigan Diabetes Research and Training Center. Some antibodies were provided by Caltag or by eBioscience to screen for cancer stem-cell markers. Human primary melanocyte cultures were provided by $M$. Soengas. Human mesenchymal stem cells were provided by Z. Wang and P. Krebsbach. E.Q. was supported by the Spanish Ministry of Education and the Marie Curie Outgoing International Fellowship from the European Commission. M.S. was supported by the Australian National Health and Medical Research Council, the Human Frontiers Science Program and Australia Post.

Author Contributions E.Q., M.S. and S.J.M. planned the project. E.Q. and M.S. performed the experiments, and analysed data with S.J.M. M.S.S. and T.M.J. obtained consent from the patients and surgically obtained many of the melanoma specimens. D.R.F. performed all pathology and diagnosed the tumours with T.M.J. T.M.J. banked the melanomas, and provided clinical information. E.Q., M.S. and S.J.M. wrote the paper.

Author Information Reprints and permissions information is available at www.nature.com/reprints. Correspondence and requests for materials should be addressed to S.J.M. (seanjm@umich.edu). 


\section{METHODS}

Cell preparation. Tumours were mechanically dissociated with a McIlwain tissue chopper (Mickle Laboratory Engineering Co.) before sequential enzymatic digestion in $200 \mathrm{U} \mathrm{ml}^{-1}$ collagenase IV (Worthington) for $20 \mathrm{~min}$ followed by $0.05 \%$ trypsin-EGTA for $5 \mathrm{~min}$, both at $37^{\circ} \mathrm{C}$. DNase $\left(50-100 \mathrm{U} \mathrm{ml}^{-1}\right)$ was added to reduce clumping of cells during digestion. Cells were filtered $(40-\mu \mathrm{m}$ cell strainer) to obtain a single-cell suspension. Dead cells and debris were reduced by density centrifugation $\left(1.1 \mathrm{~g} \mathrm{ml}^{-1}\right.$ Optiprep, Sigma) and/or magnetic bead separation (Dead Cell Removal Kit; Miltenyi Biotec) as necessary. Primary human melanocytes ${ }^{41}$ and mesenchymal stem cells ${ }^{42}$ were cultured as described. Cell labelling and sorting. All antibody labelling of cells was performed for $20 \mathrm{~min}$ on ice, followed by washing and centrifugation. Secondary antibodies were conjugated to phycoerythrin (goat anti-mouse IgG or IgM, goat anti-rat IgG or goat anti-rabbit IgG; Jackson ImmunoResearch). Primary isotype controls followed by the same secondary antibodies were used to set background. Cells were subsequently stained with directly conjugated antibodies to human CD45 (HI30-APC, BD Biosciences), human CD31 (WM59-APC, eBiosciences) and Glycophorin A (HIR2-APC, Biolegend) (for tumours obtained directly from patients) or mouse CD45 (30-F11-APC, eBiosciences), mouse CD31 (390-APC, Biolegend), Ter119 (TER-119-APC, eBiosciences) and human HLA-A,B,C (G46-2.6-FITC, BD Biosciences) (for xenograft tumours) to select live human melanoma cells and to exclude endothelial and haematopoietic cells. Cells were re-suspended in $10 \mu \mathrm{g} \mathrm{ml}^{-1}$ DAPI (Sigma) and sorted on a FACSVantage SEdual laser, three line flow cytometer or a FACSAria Cell Sorter (Becton Dickinson). After sorting, an aliquot of sorted cells was always re-analysed to check for purity, which was usually greater than 95\%. Magnetic cell separation using the CD133 Cell Isolation Kit (Miltenyi Biotec) was performed according to the manufacturer's instructions.

Identification of single melanoma cells. Sorted cells were diluted and aliquoted into $10-\mu \mathrm{l}$ microwells (Thermo Fisher Scientific). Plates were centrifuged at $450 \mathrm{~g}$ for $30 \mathrm{~s}$ and wells containing single cells were identified by phase-contrast microscopy. In control experiments, the presence of microscopically identified single cells was confirmed by Acridine Orange staining, which demonstrated a single nucleus in 90/90 cases. Cell doublets could be identified easily, were rare ( 7 of 312 wells examined) and were discarded. Wells containing single cells were transferred to a syringe containing Matrigel before injection. Cell transfer to the syringe was confirmed by observing the absence of a cell in the well it came from. Transplantation of melanoma cells. Subcutaneous injections were performed into each flank and the interscapular region of each mouse. Tumour formation was evaluated regularly by palpation of injection sites, and tumour diameters were measured with callipers. Mice were monitored for up to 32 weeks after injection. In cases where a tumour became palpable at only one injection site and was causing the mouse distress, that tumour was surgically removed to allow continued evaluation of other injection sites. Tumours were only evident at injection sites and we never observed subcutaneous metastases. To confirm this, we performed a single subcutaneous injection of 200 cells from tumour 205 into each of six NOD/SCID Il $2 \mathrm{rg}^{-1-}$ mice after mixing in Matrigel and observed only one tumour per mouse at the injection site. The presence of human melanomas was always confirmed at necropsy based on gross examination and marker expression.

Histopathology and immunostaining. Portions of melanoma tumours used in experiments were fixed in 10\% neutral buffered formalin, paraffin embedded, sectioned and stained with haematoxylin and eosin for histopathology analysis. Paraffin-embedded tumours were confirmed as melanomas by staining for S100 and HMB45 expression after quenching endogenous peroxidase activity. Binding of anti-S100 antibody (DAKO) was performed for $30 \mathrm{~min}$ at room temperature, detected by anti-rabbit secondary (30 min at room temperature) and revealed using DAB chromagen. Binding of HMB45 antibody (DAKO) was performed for $30 \mathrm{~min}$ at room temperature after antigen retrieval with proteinase K, detected using the M.O.M. immunodetection kit (Vector Laboratories) and revealed using DAB. S100- and HMB45-stained slides were counterstained with haematoxylin. For staining of sorted cells, cells from tumours were cytospun ( $18 \mathrm{~g}$ for $6 \mathrm{~min}$ ) onto slides after fixation with $4 \%$ paraformaldehyde for $5 \mathrm{~min}$ at room temperature. For immunofluorescence, slides were rinsed in PBS and blocked in goat serum solution (PBS containing 5\% goat serum, 1\% BSA and $0.3 \%$ Triton $\mathrm{X}-100$ ) for $1 \mathrm{~h}$ to reduce non-specific antibody binding. Incubation with S-100 $\beta$ antibody (Sigma, diluted 1:200 in goat serum solution) was performed overnight at $4{ }^{\circ} \mathrm{C}$, followed by secondary goat anti-mouse IgG1 Alexa 488 (Invitrogen) for $2 \mathrm{~h}$ at room temperature. Slides were counterstained with DAPI for $10 \mathrm{~min}$ at room temperature, then mounted in fluorescent mounting solution (DAKO). For detection of melanin pigment, the FontanaMasson method was used ${ }^{43}$. Slides were microwaved for 1 min in $2.5 \%$ Fontana silver nitrate solution (Sigma), before rinsing and toning with $0.2 \%$ gold chloride for $2 \mathrm{~min}$. After rinsing, slides were incubated in 5\% sodium thiosulphate (Sigma) for $2 \mathrm{~min}$, washed and nuclei were stained with DAPI. Slides were mounted in DAKO fluorescent mounting solution.

Statistics. Differences between mean times to tumour palpability were compared using unpaired $t$-tests. Tumour growth rates were determined by calculating averaged linear regression slopes of the diameters of each tumour at each time point, monitored for at least 10 days and displayed with dot points representing the mean ( \pm s.d.) of the diameters of all tumours palpable at the indicated time points.

41. Fernandez, Y. et al. Differential regulation of noxa in normal melanocytes and melanoma cells by proteasome inhibition: therapeutic implications. Cancer Res. 65, 6294-6304 (2005).

42. Wang, Z. et al. Ablation of proliferating marrow with 5-fluorouracil allows partial purification of mesenchymal stem cells. Stem Cells 24, 1573-1582 (2006).

43. Bancroft, J. D. \& Stevens, A. Theory and Practice of Histological Techniques (Churchill Livingstone, 1990). 